\title{
Finger counting and numerical cognition
}

\author{
Martin H. Fischer ${ }^{1}{ }^{*}$, Liane Kaufmann ${ }^{2}$ and Frank Domahs ${ }^{3}$ \\ 1 Department of Cognitive Science, University of Potsdam, Potsdam, Germany \\ 2 Private University for Health Sciences, Medical Informatics and Technology, Hall in Tirol, Austria \\ ${ }^{3}$ RWTH Aachen University Hospital, Aachen, Germany \\ *Correspondence: martinf@uni-potsdam.de
}

Following a recent surge of interest in finger-based number knowledge, we invited empirical and conceptual contributions to assess the feasibility of a Research Topic on this issue. We received a considerable number of submissions, many of which were further improved by constructive and interactive peer-review and ultimately appeared as part of the Research Topic "Handy numbers: Finger counting and numerical cognition." We wish to thank all authors and reviewers, as well as the publisher's support team around Meghan Hodge, for their excellent work.

This enthusiastic response from the research community confirmed our expectation that the time is ripe to consider the domain of number knowledge from the theoretical perspective of embodied cognition. This domain is particularly challenging for an embodied perspective on human cognition because mental arithmetic was thought to consist of abstract and amodal symbol manipulation. By disregarding the acquisition, implementation, or retrieval context of such knowledge, numerical cognition provided an ideal example of abstract information processing (e.g., Groen and Parkman, 1972). Yet in recent years a flurry of reports documented just such sensory and motor contributions to numerical cognition, and the contributions gathered for the present research topic on "handy numbers" provide an up-to-date survey of this development.

The published contributions make clear that there is no agreement about the relevance of finger counting for numerical cognition. For example, finger associations might not be a necessary component of number knowledge acquisition (Crollen et al., 2011),

\section{REFERENCES}

Beller, S., and Bender, A. (2011). Explicating numerical information: when and how fingers support (or hinder) number comprehension and handling. Front. Psychol. 2:214. doi: 10.3389/fpsyg.2011.00214

Bender, A., and Beller, S. (2011). Fingers as a tool for counting - naturally fixed or culturally flexible? Front. Psychol. 2:256. doi: 10.3389/fpsyg.2011.00256

Costa, A. J., Chagas, P. P., Krinzinger, H., Lonneman, J., Willmes, K., Wood, G., and Haase, V. G. (2011). A hand full of numbers: a role for offloading in arithmetics learning? Front. Psychol. 2:368. doi: 10.3389/fpsyg.2011.00368

Crollen, V., Seron, X., and Noël, M.-P. (2011). Is finger-counting necessary for the development of arithmetic abilities? Front. Psychol. 2:242. doi: 10.3389/fpsyg.2011.00242

Di Luca, S., and Pesenti, M. (2011). Finger numeral representations: more than just another symbolic code. Front. Psychol. 2:272. doi: 10.3389/fpsyg.2011.00272
Domahs, F., Klein, E., Moeller, K., Nuerk, H.-C., Yoon, B.-C., and Willmes, K. (2012). Multimodal semantic quantity representations: further evidence from Korean sign language. Front. Psychol. 2:389. doi: 10.3389/fpsyg.2011.00389

Fischer, M. H., and Brugger, P. (2011). When digits help digits: spatialnumerical associations point to finger counting as prime example of embodied cognition. Front. Psychol. 2:260. doi: 10.3389/fpsyg.2011.00260

Groen, G. J., and Parkman, J. M. (1972). A chronometric analysis of simple addition. Psychol. Rev. 79, 329-343.

Imbo, I., Vandierendonck, A., and Fias, W. (2011).Passive hand movements disrupt adults' counting strategies. Front. Psychol. 2:201. doi: 10.3389/fpsyg.2011.00201

Kaufmann, L., Pixner, S., and Göbel, S. M. (2011). Finger usage and arithmetic in adults with math difficulties: evidence from a case report. Front. Psychol. 2:254. doi: 10.3389/fpsyg.2011.00254

Klein, E., Moeller, K., Willmes, K., Nuerk, H.-C., and Domahs, F. (2011). The

they might merely reflect immature retrieval strategies (Kaufmann et al., 2011), and some aspects of finger-based number representation might actually hinder the initial learning process (Beller and Bender, 2011). Nevertheless, all empirical contributions to this research topic support a role of fingers in numerical cognition: Spatial-numerical associations, previously attributed to reading habits, may at least partly have their origin in finger counting routines (Fischer and Brugger, 2011; Riello and Rusconi, 2011). They are prevalent in finger counting systems of many cultures (Previtali et al., 2011; Domahs et al., 2012), affect a wide range of behaviors (Fischer and Brugger, 2011), depend on hand orientation (Previtali et al., 2011), and possibly on finger gnosia (Costa et al., 2011; Reeve and Humberstone, 2011). Furthermore, finger usage and finger-based number representations may vary considerably according to cultural influences (Bender and Beller, 2011; Domahs et al., 2012). Mental addition is selectively impaired by passive hand movements (Imbo et al., 2011) and shows sub-base five effects that can be attributed to hand-based representations (Klein et al., 2011). Addition also activates finger-related cortical structures (Krinzinger et al., 2011).

Findings such as these highlight the special status of finger representations in numerical cognition (Di Luca and Pesenti, 2011) and require a conceptual rethinking. This can begin by aligning educational and neuroscientific perspectives (Moeller et al., 2011) or by contextualizing them within the embodied cognition framework (Fischer and Brugger, 2011).

influence of implicit hand-based representations on mental arithmetic. Front. Psychol. 2:197. doi: 10.3389/ fpsyg.2011.00197

Krinzinger, H., Koten, J. W., Horoufchin, H., Kohn, N., Arndt, D., Sahr, K., Konrad, K., and Willmes, K. (2011). The role of finger representations and saccades for number processing: an fMRI study in children. Front. Psychol. 2:373. doi: 10.3389/fpsyg.2011.00373

Moeller, K., Martignon, L., Engel, J., and Nuerk, H.-C. (2011). Effects of finger counting on numerical development - the opposing views of neurocognition and mathematics education. Front. Psychol. 2:328. doi: 10.3389/ fpsyg. 2011.00328

Previtali, P., Rinaldi, L., and Girelli, L. (2011). Nature or nurture in finger counting: a review on the determinants of the direction of numberfinger mapping. Front. Psychol. 2:363. doi: $10.3389 /$ fpsyg.2011.00363

Reeve, R., and Humberstone, J. (2011). Five- to 7-year-olds' finger gnosia and calculation abilities. Front. Psychol. 2:359. doi: 10.3389/ fpsyg.2011.00359

Riello, M., and Rusconi, E. (2011). Unimanual SNARC effect: hand matters. Front. Psychol.2:372. doi: 10.3389/ fpsyg.2011.00372

Received: 22 March 2012; accepted: 26 March 2012; published online: 12 April 2012.

Citation: Fischer MH, Kaufmann L and Domahs F (2012) Finger counting and numerical cognition. Front. Psychology 3:108. doi: 10.3389/fpsyg.2012.00108

This article was submitted to Frontiers in Cognition, a specialty of Frontiers in Psychology.

Copyright $\odot 2012$ Fischer, Kaufmann and Domahs. This is an open-access article distributed under the terms of the Creative Commons Attribution Non Commercial License, which permits non-commercial use, distribution, and reproduction in other forums, provided the original authors and source are credited. 